\title{
Sequential formation of two branched intermediates during protein splicing of class three inteins
}

\author{
Kazuo Tori ${ }^{1,2} \cdot$ Francine Perler $^{1,3,4}$
}

Received: 15 July 2016 / Accepted: 24 September 2016 / Published online: 4 October 2016

(C) The Author(s) 2016. This article is published with open access at Springerlink.com

\begin{abstract}
Inteins are the protein equivalent of introns. They are seamlessly removed during post-translational maturation of their host protein (extein). Inteins from extremophiles played a key role in understanding intein-mediated protein splicing. There are currently three classes of inteins defined by catalytic mechanism and sequence signatures. This study demonstrates splicing of three class 3 miniinteins: Burkholderia vietnamiensis G4 Bvi IcmO intein, Mycobacterium smegmatis MC2 155 Msm DnaB-1 intein and Mycobacterium leprae strain TN Mle DnaB intein. $B$. vietnamiensis has a broad ecological range and remediates trichloroethene. M. smegmatis is a biofilm forming soil bacteria. Although other intein classes have only a single branched intermediate at the C-terminal splice junction, the class 3 intein reaction pathway includes two branched intermediates. The class 3 specific branched intermediate is formed by an internal cysteine, while the C-terminal branch
\end{abstract}

Communicated by H. Atomi.

This article is part of a special feature based on the 11th International Congress on Extremophiles held in Kyoto, Japan, September 12-16, 2016.

Francine Perler

PerlsOfWisdom@icloud.com

Kazuo Tori

hiris5211@yahoo.co.jp

1 New England Biolabs, Inc., Ipswich, MA 01938, USA

2 Present Address: Takara Bio USA, Inc., 1290 Terra Bella Ave., Mountain View, CA 94043, USA

3 Present Address: Perls of Wisdom Biotech Consulting, Brookline, MA 02446, USA

4 Department of Molecular Biophysics and Biochemistry, Yale University, New Haven, CT 06520, USA intermediate is at a serine or threonine in all class 3 inteins except the Bvi IcmO intein, where it is a cysteine. This latter cysteine was unable to compensate for mutation of the class 3-specific internal catalytic cysteine despite the Bvi IcmO intein having an N-terminal splice junction naturally tuned for a cysteine nucleophile, demonstrating the mandatory order of branch intermediates in class 3 inteins.

Keywords Intein $\cdot$ Protein splicing $\cdot$ Branched intermediate $\cdot$ Enzyme mechanism $\cdot$ Cysteine

$\begin{array}{ll}\text { Abbreviations } & \text { Amino acid } \\ \text { aa } & \text { Branched intermediate } \\ & \text { Branched intermediate with } \\ \mathrm{BI}^{\mathrm{F}} & \mathrm{Cys}^{\mathrm{F}: 4} \text { as the branch point } \\ & \text { Branched intermediate with } \\ & \text { the }+1 \text { (G8) amino acid as the } \\ \mathrm{BI}^{\mathrm{G}} & \text { branch point } \\ & \text { Maltose binding protein } \\ \mathrm{M} \text { or MBP } & \text { Paramyosin } \Delta \text { Sal fragment } \\ \mathrm{P} & \text { Intein } \\ \mathrm{I} & \text { Model precursors with MBP } \\ \mathrm{MIP}, \mathrm{MSP}, \mathrm{MLP} \text { and MVP } & \text { and P flanking either any } \\ & \text { intein, the Msm DnaB-1 } \\ & \text { intein, the Mle DnaB intein } \\ & \text { or the Bvi IcmO intein, } \\ & \text { respectively }\end{array}$

\section{Introduction}

Inteins are protein splicing elements that are removed from precursor proteins by a self-catalytic mechanism. Inteins from extremophiles were key to defining all three currently 
known mechanisms of intein-mediated protein splicing (Brace et al. 2010; Eryilmaz et al. 2014; Mills et al. 2014; Southworth et al. 2000; Tori et al. 2010; Volkmann and Mootz 2013; Xu et al. 1994; Xu and Perler 1996). Over 500 intein genes have been identified in numerous archaea, eubacteria, single cell eukaryotes and viruses (Perler 2002), and the numbers are growing rapidly with the explosion of archaeal and bacterial genome sequences. Inteins disrupt the function of their host protein (termed an extein) and thus protein splicing is required for survival if the intein is present in an essential protein (Dalgaard et al. 1997; Novikova et al. 2014; Perler 2002). The intein and extein are translated as a single, fused precursor protein. During post-translational maturation, the intein removes itself from this precursor while joining the flanking extein fragments with a native peptide bond. No external enzyme or cofactor is required. The majority of inteins are bifunctional enzymes that have a homing endonuclease domain as well as a protein splicing domain. The homing endonuclease is responsible for lateral transmission of intein genes, making them parasitic mobile genetic elements (Barzel et al. 2011; Novikova et al. 2014). Mini-inteins do not have an endonuclease domain, but retain the core protein splicing domain. Modern day mini-inteins are thought to be the descendents of inteins that lost their homing endonuclease domain (Barzel et al. 2011; Novikova et al. 2014).

Very few inteins have been characterized biochemically or even shown to be functional (Perler 2002). In this study we characterized the activity of three mini-inteins: the Burkholderia vietnamiensis G4 Bvi IcmO intein (Nordberg et al. 2014; Perler 2002), the Mycobacterium smegmatis MC2 155 Msm DnaB-1 intein (Mohan et al. 2015; Perler 2002), and the Mycobacterium leprae TN Mle DnaB intein (Eiglmeier et al. 1993; Perler 2002). DnaB is a replicative helicase in bacteria. The function of IcmO has yet to be established. The Bvi IcmO gene is present on a B. vietnamiensis plasmid, not the main chromosome (Nordberg et al. 2014). B. vietnamiensis $G 4$ has a broad ecological range, fixes nitrogen, remediates trichloroethene and is found in the lungs of Cystic Fibrosis patients (Nordberg et al. 2014). $M$. smegmatis is a soil bacteria that forms biofilms and $M$. leprae is a slow growing human pathogen.

Inteins are currently divided into three classes based on differences in their protein splicing mechanism (Fig. 1) and conserved signature sequences (Tori et al. 2010). All inteins have at least four conserved motifs (Blocks A, B, $\mathrm{F}$ and G) in the splicing domain (Perler 2002; Perler et al. 1997; Pietrokovski 1994, 1998). Amino acids (aa) within these conserved motifs are numbered using the block designation and the position within the block, separated by a colon (Perler 2002; Tori et al. 2010). For example, the fourth amino acid in Block F is referred to as F:4. Several amino acids in these conserved motifs are present at or near the intein active site where they directly act as nucleophiles and facilitating residues for catalysis or they assist in proper packing of the intein active site to align catalytic residues (Brace et al. 2010; Eryilmaz et al. 2014; Kawasaki et al. 1997; Mills et al. 2014; Romanelli et al. 2004; Southworth et al. 2000; Tori et al. 2010; Volkmann and Mootz 2013; Xu et al. 1994; Xu and Perler 1996). Catalytically important residues common to all classes of inteins include $\mathrm{His}^{\mathrm{B}: 10}$ (essential for $\mathrm{N}$-terminal splice junction reactions), the intein penultimate $\mathrm{His}^{\mathrm{G}: 6}$ (assists in C-terminal splice junction reactions), the intein $\mathrm{C}$-terminal $\mathrm{Asn}^{\mathrm{G}: 7}$ (responsible for $\mathrm{C}$-terminal splice junction cleavage during resolution of the Block $\mathrm{G}$ branched intermediate, $\mathrm{BI}^{\mathrm{G}}$ ) and $\mathrm{Ser}^{+1}, \mathrm{Cys}^{+1}$ or $\mathrm{Thr}^{+1}$ [the $\mathrm{G}: 8$ residue, responsible for $\mathrm{BI}^{\mathrm{G}}$ formation and conversion of a (thio)ester bond to an amide peptide bond between the ligated exteins]. Each intein has a larger set of facilitating residues that are tuned to its specific set of nucleophiles (Eryilmaz et al. 2014; Kawasaki et al. 1997; Mills et al. 2014; Romanelli et al. 2004; Tori et al. 2010; Volkmann and Mootz 2013; Wu et al. 2014; Xu and Perler 1996). Moreover, amino acids in the extein, especially proximal ones, influence protein splicing by directly participating in catalysis or by affecting the architecture of the intein active site (Amitai et al. 2009; Cheriyan et al. 2013, 2014; Chong et al. 1997; Eryilmaz et al. 2014; Iwai et al. 2006; Liu et al. 2014; Muona et al. 2010; Perler et al. 1994, 1997; Southworth et al. 1999; Volkmann and Mootz 2013; Xu et al. 1994; Xu and Perler 1996). Class 3 inteins have an additional signature sequence (Table 1) consisting of a dispersed triplet: $\operatorname{Trp}^{\mathrm{B}: 12}, \mathrm{Cys}^{\mathrm{F}: 4}$, and $\mathrm{Thr}^{\mathrm{G}: 5}$ (Tori et al. 2010). Previous studies indicated that mutation of $\mathrm{Thr}^{\mathrm{G}: 5}$ can have a minimal effect, while mutation of $\operatorname{Trp}^{\mathrm{B}: 12}$ more significantly impairs splicing, and mutation of $\mathrm{Cys}^{\mathrm{F}: 4}$ totally blocks splicing (Brace et al. 2010; Tori et al. 2010; Tori and Perler 2011). Another feature of class 3 inteins is $\mathrm{Ser}^{+1}$ or $\mathrm{Thr}^{+1}$ at the $\mathrm{G}: 8$ position instead of $\mathrm{Cys}^{+1}$, which dominates this position in other intein classes (Brace et al. 2010; Perler 2002; Southworth et al. 2000; Tori et al. 2010; Tori and Perler 2011). The Bvi IcmO intein is the only currently known class 3 intein with $\mathrm{Cys}^{+1}$.

Protein splicing requires two or three catalyzed nucleophilic displacement reactions, depending on the intein class, followed by a spontaneous acyl rearrangement that results in a peptide bond between the ligated exteins (Fig. 1). Inteins perform these reactions by functioning as single turnover enzymes. Single turnover enzymes use the same methods as traditional enzymes to achieve catalysis, but do not act on multiple substrates. Although an intein was originally defined as the sequence that is removed from a precursor protein (Perler et al. 1994), when discussing the enzymatic properties of an intein we include the +1 aa (G:8) because it actively participates in splicing. The majority of inteins belong to class 1 , which splices in four 


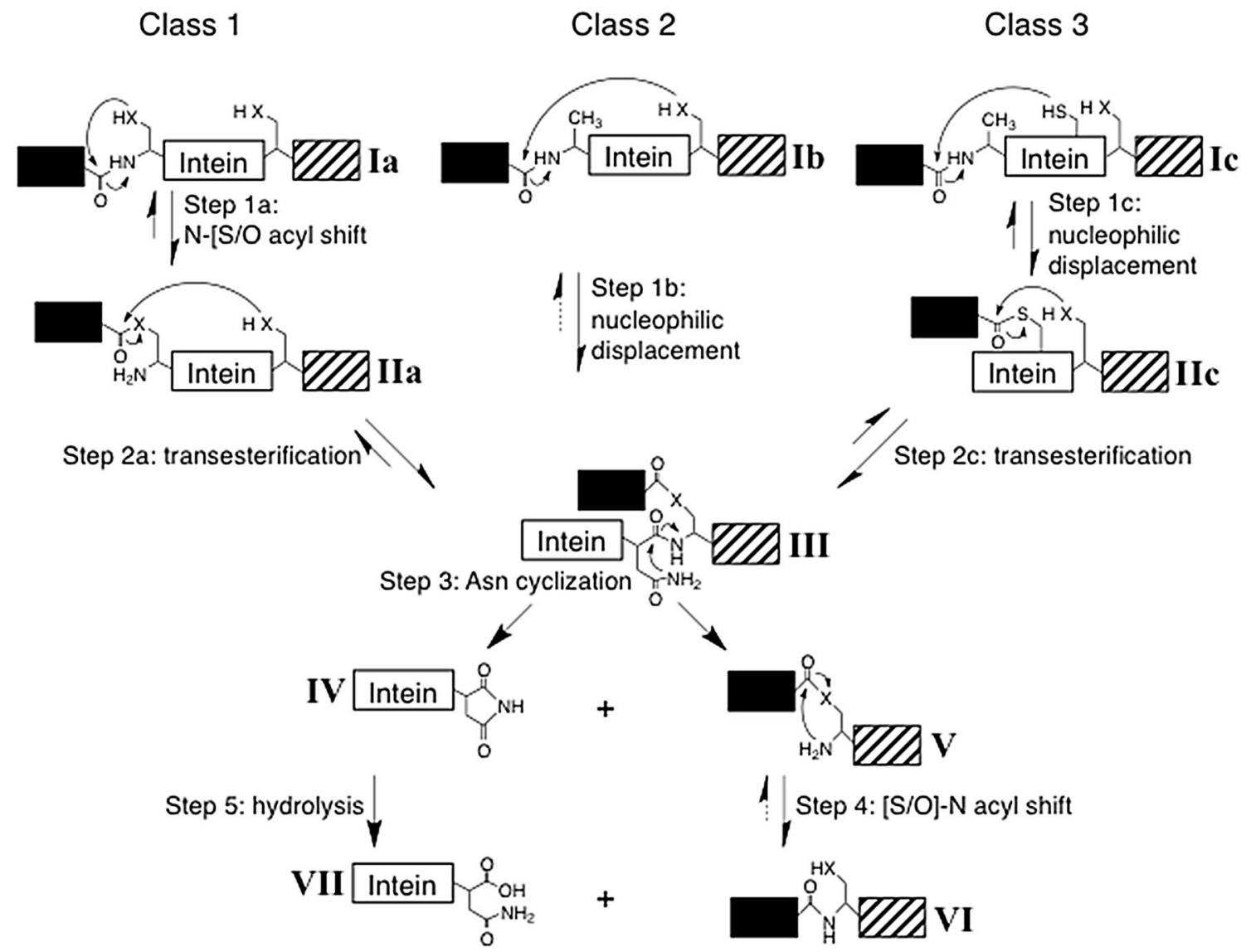

Fig. 1 Intein-mediated protein splicing mechanisms. The majority of inteins follow the class 1 intein-mediated protein splicing mechanism, which consists of four coordinated nucleophilic displacements and requires $\mathrm{Ser}^{1}, \mathrm{Thr}^{1}$ or $\mathrm{Cys}^{1}$ as the intein $\mathrm{N}$-terminal residue. Step 1a results in a linear (thio)ester intermediate and step 2a results in $\mathrm{BI}^{\mathrm{G}}$ with $\mathrm{Cys}^{+1}, \mathrm{Ser}^{+1}$ or $\mathrm{Thr}^{+1}$ as the branch point. Class 2 and 3 inteins do not require an intein $\mathrm{N}$-terminal nucleophile. Class 2 inteins directly form $\mathrm{BI}^{\mathrm{G}}$ when the +1 residue attacks the $\mathrm{N}$-terminal splice junction peptide bond. Class 3 inteins use a conserved Cys at Block $\mathrm{F}$ position $4\left(\mathrm{Cys}^{\mathrm{F}: 4}\right)$ to initiate protein splicing resulting in formation of the class-specific $\mathrm{BI}^{\mathrm{F}}$. Once $\mathrm{BI}^{\mathrm{G}}$ is formed, the remaining reactions are the same for all inteins. The acyl shift in step 4 is rapid and spontaneous. Step 5 is also spontaneous, but is often slow. Solid arrows represent steps that have been experimentally verified while dashed arrows represent theoretical steps. Note that steps 1 and 2 are reversible; the forward reactions are driven by kinetic rates, equilibrium positions toward the forward reaction, and substrate/intermediate elimination as the protein moves toward the final products, among other factors. Intein residues and flanking extein residues that assist these reactions are not shown, nor are tetrahedral intermediates. ' $\mathrm{X}$ ' represents the sulfur or oxygen atom in the side chain of Cys, Ser or $\mathrm{Thr}$

Table 1 Intein conserved motifs

\begin{tabular}{llccr} 
Intein & Block A & Block B & Block & Block G \\
\hline & 1 & 10 & 4 & $67 / 8$ \\
Bvi Icmo & /PQPLHSLVRMADG & GRSVEAARVHHWPV & PARCLVVADEMHCYI & HDIVTHN $/ \underline{\mathrm{C}}$ \\
Mle DnaB & /ALALDTPLPTPTG & GTVIVADAQHQWPT & PVRCVEVDNAAHLYL & GMVPTHN $/ \underline{\mathrm{S}}$ \\
Msm DnaB-1 & /ALALDTPLPTPSG & GTAIVADAQHQWPT & PVRCVEVDNPEHLYL & GMVPTHN $/ \underline{\mathrm{S}}$
\end{tabular}

The sequence of each motif is listed with the intein class 3 signature positions marked by an asterisk. The slash denotes each splice junction. The position number within each block of selected catalytically important amino acids is listed. The class 3 specific $\mathrm{BI}^{\mathrm{F}}$ branch point $(\mathrm{F}: 4)$ is shaded gray and the $\mathrm{BI}^{\mathrm{G}}$ branch point common to all inteins $(\mathrm{G}: 8$ or +1$)$ is underlined 
well-known steps (Eryilmaz et al. 2014; Mills et al. 2014; Perler 2002; Volkmann and Mootz 2013). Class 1 is defined by an intein $\mathrm{N}$-terminal $\mathrm{Ser}^{1}$, $\mathrm{Thr}^{1}$ or $\mathrm{Cys}^{1}$ that forms a linear (thio)ester intermediate (IIa, Fig. 1) prior to forming $\mathrm{BI}^{\mathrm{G}}$ (III). Class 2 inteins do not have a Cys ${ }^{1}, \mathrm{Thr}^{1}$ or Ser ${ }^{1}$ (Perler 2002; Southworth et al. 2000). Instead, the +1 aa directly attacks the peptide bond at the N-terminal splice junction (step 1b) to yield a standard $\mathrm{BI}^{\mathrm{G}}$ (Southworth et al. 2000). Only class 2 inteins can perform step 1b. Class 3 inteins are similar to class 2 inteins since an intein $\mathrm{N}$-terminal Cys ${ }^{1}, \mathrm{Thr}^{1}$ or $\mathrm{Ser}^{1}$ is not required for splicing (Brace et al. 2010; Perler 2002; Tori et al. 2010; Tori and Perler 2011). However, class 3 inteins initiate splicing when the class-specific conserved $\mathrm{Cys}^{\mathrm{F}: 4}$ attacks the peptide bond at the $\mathrm{N}$-terminal splice junction (step 1c) resulting in the formation of the class 3-specific Block F BI $\left(\mathrm{BI}^{\mathrm{F}}\right.$, IIc) (Brace et al. 2010; Tori et al. 2010; Tori and Perler 2011). BI ${ }^{\mathrm{G}}$ (III) is then formed by a transesterification reaction (step 2c). All inteins follow the same pathway for $\mathrm{BI}^{\mathrm{G}}$ resolution and formation of the peptide bond between the exteins.

This study examined whether three mini-inteins lacking an N-terminal $\mathrm{Ser}^{1}, \mathrm{Thr}^{1}$ or $\mathrm{Cys}^{1}$ are functional and the mechanism by which they splice. For the first time we were able to test whether there is a mandatory order of BI formation in a class 3 intein that naturally has $\mathrm{Cys}^{+1}$ and an $\mathrm{N}$-terminal splice junction already tuned for attack by a Cys nucleophile.

\section{Materials and methods}

\section{Cloning, mutagenesis, and protein expression}

All clones were sequenced by the New England Biolabs core facility. The genes for the Msm DnaB-1 and Mle DnaB inteins with flanking DnaB extein sequences and appropriate restriction enzyme sites were synthesized by Invitrogen (Carlsbad, CA, USA). The gene encoding the Bvi IcmO intein with flanking $\mathrm{IcmO}$ extein residues was amplified by PCR from $B$. vietnamiensis $G 4$ genomic DNA using Phusion DNA polymerase and primers containing $X h o I$ and SpeI restriction enzyme sites. The Msm DnaB-1 and Mle DnaB intein precursors included native DnaB flanking residues Phe-Gly-Val-Gly-Lys (N-extein) and Ser-Thr-LeuGly-Leu (C-extein), while the Bvi IcmO intein precursor included native flanking IcmO residues Ala-Arg-Ser-LeuGly-Phe (N-extein) and Cys-Ile-Thr-Phe-Ala (C-extein). The DNAs were digested by XhoI and SpeI, agarose gel purified and ligated into pMP1 (Southworth et al. 2000; Tori et al. 2010) previously digested with the same enzymes. This resulted in pM묘 with the Msm DnaB-1 intein, pMLP

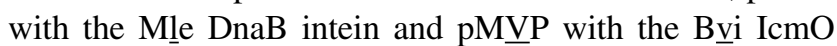
intein, where the intein was flanked with the Escherichia coli maltose-binding protein ( $\mathrm{M}$ or $\mathrm{MBP})$ and the $\Delta S a l$ fragment of Dirofilaria immitis paramyosin (P).

By convention, amino acids in the intein are numbered beginning with the intein $\mathrm{N}$-terminus and residues in the $\mathrm{C}$-extein are numbered similarly, but contain a plus sign to denote the C-extein. His ${ }^{\mathrm{B}: 10}, \operatorname{Trp}^{\mathrm{B}: 12}, \mathrm{Cys}^{\mathrm{F}: 4}$ and $\mathrm{Asn}^{\mathrm{G}: 7}$ are, respectively, $\mathrm{His}^{65}, \operatorname{Trp}^{67}$, Cys ${ }^{118}$ and $\mathrm{Asn}^{139}$ in the Msm DnaB-1 intein, $\mathrm{His}^{65}, \mathrm{Trp}^{67}$, Cys ${ }^{124}$ and $\mathrm{Asn}^{145}$ in the Mle DnaB intein and His ${ }^{65}, \operatorname{Trp}^{67}$, Cys ${ }^{122}$ and $\mathrm{Asn}^{142}$ in the Bvi IcmO intein (Perler 2002).

All mutations were made using the Phusion site-directed mutagenesis kit (New England Biolabs) with primers that introduced the desired mutation. For protein expression, freshly transformed E.coli NEB Turbo cells were grown in LB media containing $100 \mu \mathrm{g} / \mathrm{ml}$ ampicillin at $37^{\circ} \mathrm{C}$ until reaching an $\mathrm{OD}_{600}$ of $\sim 0.5$ and then induced with $0.4 \mathrm{mM}$ IPTG at room temperature, $30{ }^{\circ} \mathrm{C}$ and $37{ }^{\circ} \mathrm{C}$ for $2 \mathrm{~h}$ or at $15{ }^{\circ} \mathrm{C}$ overnight. Protein splicing was assessed using soluble lysates after electrophoresis in 4-20\% SDS-PAGE (Invitrogen, Carlsbad, CA, USA). Proteins were detected by either Simply Blue Safe Stain (Invitrogen) or fluorescent Western Blot as described previously (Cheriyan et al. 2013; Southworth et al. 2000; Tori and Perler 2011). Briefly, nitrocellulose filters were concurrently probed with mouse anti-MBP sera and rabbit anti-paramyosin sera, and then developed concurrently with IR-Dye 680 anti-mouse secondary antibody (green) or IR-Dye 800 anti-rabbit secondary antibody (red) (LI-COR, Lincoln, NE, USA).

\section{Purification and characterization of branched intermediates}

BIs of Msm DnaB-1 and Mle DnaB inteins with Asn ${ }^{\mathrm{G}: 7}$ mutated to alanine in MVP and MLP were purified by affinity chromatography over amylose resin. The $\mathrm{pH}$ of an aliquot of each purified BI was changed by addition of sodium phosphate buffer. Samples were incubated overnight at room temperature in the absence or presence of $50 \mathrm{mM}$ DTT. Time zero $\left(T_{0}\right)$ samples were heated at $100{ }^{\circ} \mathrm{C}$ for $5 \mathrm{~min}$ in SDS-PAGE sample buffer (New England Biolabs) without incubation. An aliquot of purified MLP BI was denatured by adding solid urea to a final concentration of $8 \mathrm{M}$. The $\mathrm{pH}$ of the denatured sample was checked prior to subsequent treatment as above for native samples.

\section{Results}

\section{Splicing of three mini-inteins in vivo}

DNAs encoding the Msm DnaB-1 (139 aa) and Mle DnaB (145 aa) mini-inteins along with $5 \mathrm{DnaB}$ extein residues flanking the intein on each side (Perler 2002) were 


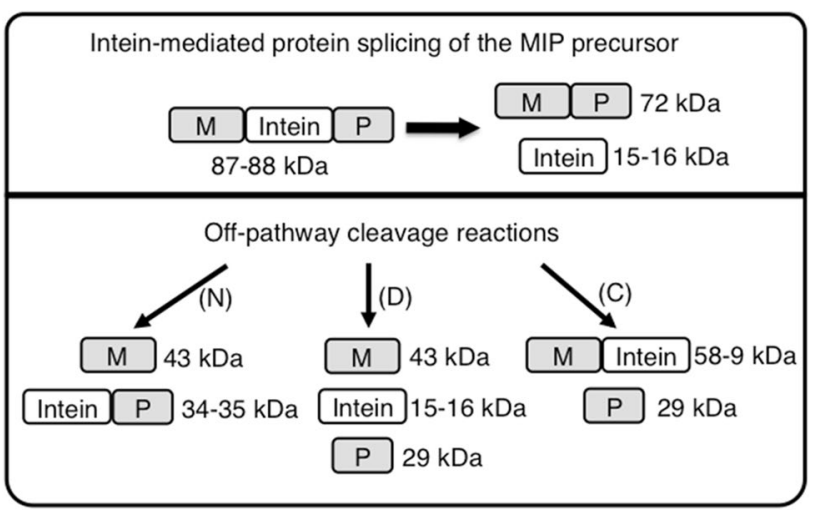

Fig. 2 Splicing and cleavage schemes for the MIP precursor with either the Bvi IcmO, Mle DnaB or Msm DnaB-1 inteins. Off-pathway cleavage reactions can occur at the $\mathrm{N}$-terminal splice junction $(\mathrm{N})$, the C-terminal splice junction (C) or both splice junctions (D). Branched intermediate decay yields the same products as direct $\mathrm{N}$-terminal splice junction cleavage $(\mathrm{N})$

synthesized and cloned in the MIP model precursor system (Xu et al. 1994) between the E. coli maltose binding protein (MBP or $\mathrm{M}$ ) and the D. immitis paramyosin $\Delta$ Sal fragment (P) generating precursors MSP and MLP, respectively. The coding sequence for the Bvi IcmO intein (142 aa) was amplified by PCR with flanking IcmO extein residues and likewise cloned into MIP generating MVP. Splicing of these model precursors (Fig. 2) results in production of MP (72 kDa) plus free intein (I, 14.7-16.0 kDa). Off-pathway cleavage reactions would result in production of $\mathrm{M}(43 \mathrm{kDa})$ and IP (34-35 kDa) after N-terminal cleavage or BI decay, and MI (58-59 kDa) and $\mathrm{P}(29 \mathrm{kDa})$ after C-terminal cleavage.

Splicing in model systems can vary at different temperatures due to differences in expression rates, precursor folding and aggregation. Therefore, in vivo splicing activity of all three inteins was examined after each precursor was expressed in E. coli at $37{ }^{\circ} \mathrm{C}, 30^{\circ} \mathrm{C}$ and room temperature for $2 \mathrm{~h}$ or at $15{ }^{\circ} \mathrm{C}$ overnight. Msm DnaB-1 and Mle DnaB inteins yielded spliced products only at $15{ }^{\circ} \mathrm{C}$ (Fig. 3 and data not shown). MVP spliced poorly with less than half of the MVP precursor converted to spliced product at all temperatures tested (Fig. 3 and data not shown). These results demonstrate that all three mini-inteins are active, although the degree of splicing in these model precursors varied with the intein. Low levels of spliced product generally reflect misfolding of model precursors, especially when the precursor accumulates as a nonreactive component.

\section{Dissecting the splicing pathway by mutation of conserved amino acids}

Although all three inteins have the class 3 signature motif (Table 1), they can potentially splice by either the class 2

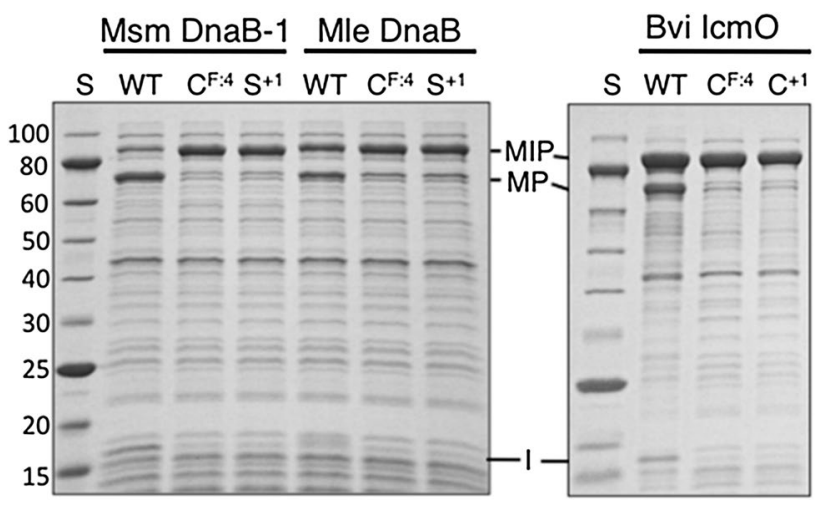

Fig. 3 Splicing of wild type and mutant inteins in model precursors. MSP (Mle DnaB intein) and MLP (Msm DnaB-1 intein) were expressed at $15{ }^{\circ} \mathrm{C}$ and MVP (Bvi IcmO intein) was expressed at $30{ }^{\circ} \mathrm{C}$. In vivo splicing of soluble proteins was examined after electrophoresis in SDS-PAGE followed by staining with Simply Blue Safe Stain. Residues mutated to alanine are listed above each lane. Lane $S$ contains the NEB 10-250 kDa Protein Ladder with molecular masses $(\mathrm{kDa})$ listed on the left. MIP precursor with the indicated inteins, $M P$ spliced product, $I$ excised intein, $W T$ wild type intein

or class 3 mechanism. Mutation of $\mathrm{Cys}^{\mathrm{F}: 4}$ can distinguish between these splicing mechanisms because it blocks the first step in the class 3 splicing pathway, but not in the class 2 pathway (Fig. 1). No splicing or cleavage was observed in MLP, MSP or MVP after substitution of $\mathrm{Cys}^{\mathrm{F}: 4}$ with alanine when analyzed by Simply Blue Safe Stained SDS-PAGE or florescent Western Blot (Fig. 3 and data not shown). Only precursor was observed when $\mathrm{His}^{\mathrm{B}: 10}$ or $\operatorname{Trp}^{\mathrm{B}: 12}$ was mutated to alanine in all three inteins, which is consistent with their importance for splicing of class 3 inteins. Nonconservative substitution of $\mathrm{Ser}^{+1}$ or $\mathrm{Cys}^{+1}$ to alanine yielded unreacted precursor in Simply Blue Safe Stained SDS-PAGE and by florescent Western Blot analysis (Fig. 3 and data not shown). However, splicing was observed after the conservative substitution of $\mathrm{Ser}^{+1}$ with Cys in MSP and MLP, while substitution of $\mathrm{Cys}^{+1}$ with Ser blocked splicing and yielded only unreacted MVP precursor as analyzed in florescent Western Blots and stained SDS-PAGE (data not shown). These results are consistent with data from many other inteins where cysteine could substitute for a catalytic serine or threonine, but serine could not substitute for a catalytic cysteine; these effects are attributed to differences in $\mathrm{pKa}$ and the greater need to activate serine or threonine side chain hydroxyls to increase their nucleophilicity compared to cysteine side chain thiols (Eryilmaz et al. 2014; Mills et al. 2014; Volkmann and Mootz 2013). Such differences in reactivities and overall amino acid size are factors contributing to the tuning of an enzyme to its specific catalytic residues. Taken together, the mutation data indicate that all three mini-inteins follow the class 3 splicing pathway (Fig. 1). 


\section{In vitro analysis of the Msm DnaB-1 and Mle DnaB intein branched intermediates}

$\mathrm{Asn}^{\mathrm{G}: 7}$ to alanine mutations in MSP and MLP resulted in BI accumulation (Fig. 4), which is normally seen as a slowly migrating band in SDS-PAGE compared to the initial precursor. These results are consistent with the role of $\mathrm{Asn}^{\mathrm{G}: 7}$ in branch resolution (step 3, Fig. 1). The nature of the BIs that accumulated in vivo was then examined
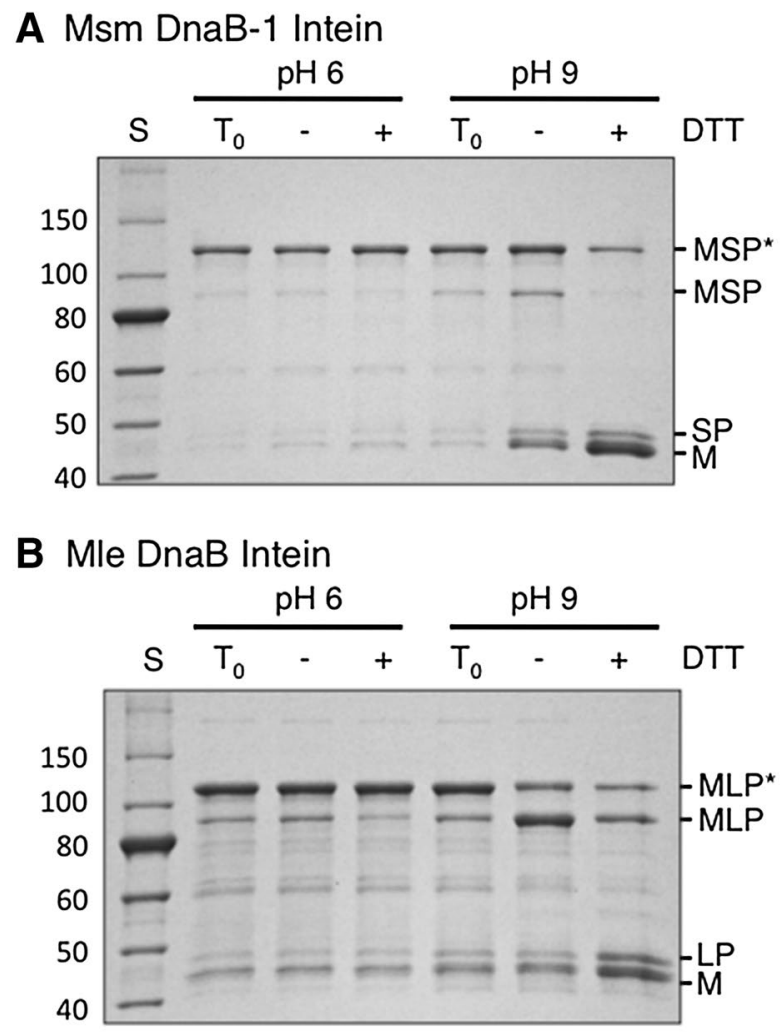

\section{Differential lability of thioesters vs. esters}

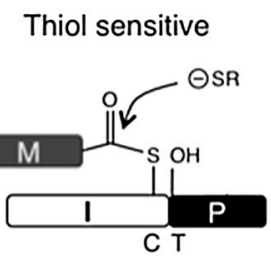

Block F Branched Intermediate

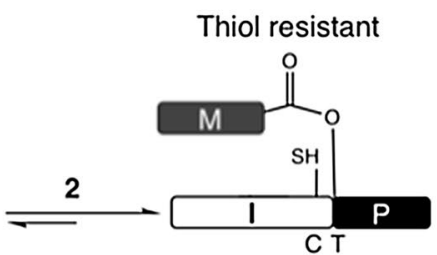

Block G Branched Intermediate
Fig. 4 Investigation of MSP and MLP branched intermediates in vitro. MSP (a) and MLP (b) BIs accumulated in vivo when the intein C-terminal Asn ${ }^{\mathrm{G}: 7}$ was mutated to alanine. Purified BIs (MSP* or MLP*) were incubated in vitro at $\mathrm{pH} 6$ or 9 in the absence $(-)$ or presence $(+)$ of $50 \mathrm{mM}$ DTT at room temperature. Time zero samples $\left(T_{0}\right)$ were not incubated in vitro. The SDS-PAGE gel was stained with Simply Blue Safe Stain. Lane S NEB 10-250 kDa Protein Ladder with molecular masses $(\mathrm{kDa})$ listed on the left of the gels. $\mathbf{c}$ Step 2 in the splicing pathway of class 3 inteins reversibly converts $\mathrm{BI}^{\mathrm{F}}$ to $\mathrm{BI}^{\mathrm{G}}$. Under the conditions of these experiments, thiol reagents can attack a thioester bond, but not an ester bond in vitro. Purified BI samples from MSP and MLP after $\mathrm{Asn}^{\mathrm{G}: 7}$ to alanine substitution were incubated overnight at room temperature at either $\mathrm{pH} 6$ or $\mathrm{pH} 9$ in the presence or absence of $50 \mathrm{mM}$ DTT (Fig. 4). The BIs were stable at $\mathrm{pH}$ 6 under all conditions tested. At $\mathrm{pH} \mathrm{9,} \mathrm{in} \mathrm{the} \mathrm{presence} \mathrm{of}$ DTT the BIs decayed to form M $+\mathrm{SP}$ or LP, while in the absence of DTT the BIs partially reverted back to MSP or MLP precursors. Stability was also tested with denatured MLP BI. No change was observed upon incubation of the denatured MLP BI sample overnight at room temperature with $50 \mathrm{mM}$ DTT at either $\mathrm{pH}$ (data not shown), indicating that thiol induced BI decay requires a properly folded protein and that the steady state BI molecules do not have detectable amounts of a thiol sensitive bond.

\section{Characterization of catalytically important cysteines in the Bvi IcmO intein}

The Bvi IcmO intein is the only currently identified class 3 intein with $\mathrm{Cys}^{+1}$ and thus both $\mathrm{BI}^{\mathrm{F}}$ and $\mathrm{BI}^{\mathrm{G}}$ have a thioester linkage. Mutation of the Bvi IcmO intein $\mathrm{Asn}^{\mathrm{G}: 7} \mathrm{did}$ not result in BI accumulation in vivo and instead yielded $\mathrm{N}$-terminal splice junction cleavage products $(\mathrm{M}+\mathrm{VP})$. This is consistent with previous studies that demonstrated in vivo lability of BI thioester linkages (Brace et al. 2010; Mills et al. 2014; Southworth et al. 2000; Tori et al. 2010; Tori and Perler 2011; Volkmann and Mootz 2013).

The Bvi IcmO intein provides a unique opportunity to examine any potential competition between the catalytic cysteines at F:4 and G:8 (+1), and to determine if $\mathrm{Cys}^{+1}$ can directly attack an $\mathrm{N}$-terminal splice junction that is already tuned for cleavage by a cysteine. Single alanine substitutions of $\mathrm{Cys}^{\mathrm{F}: 4}$ or $\mathrm{Cys}^{+1}$ yielded only unreacted precursor in both stained gels and fluorescent Western Blots (Fig. 3 and data not shown). However, when alanine substitutions of each cysteine were combined with alanine substitutions of $\mathrm{Asn}^{\mathrm{G}: 7}$, the $\mathrm{Cys}^{+1}$ double mutant produced N-terminal cleavage products while the $\mathrm{Cys}^{\mathrm{F}: 4}$ double mutant did not, as assayed in stained SDS-PAGE and confirmed in the more sensitive fluorescent Western Blot (Fig. 5). Further experimentation is necessary to determine why cleavage products were not observed with the single MVP $\mathrm{Cys}^{+1}$ mutant, especially structural studies of wild type and mutant inteins in MVP. It is possible that the Asn ${ }^{\mathrm{G}: 7}$ mutation opens up the active site for in vivo cleavage of the thioester linkage in $\mathrm{BI}^{\mathrm{F}}$.

\section{Discussion}

The Msm DnaB-1, Mle DnaB and Bvi IcmO inteins should be class 3 inteins based on their sequence signatures (Tori et al. 2010) and phylogenetic analysis (Tori and Perler 


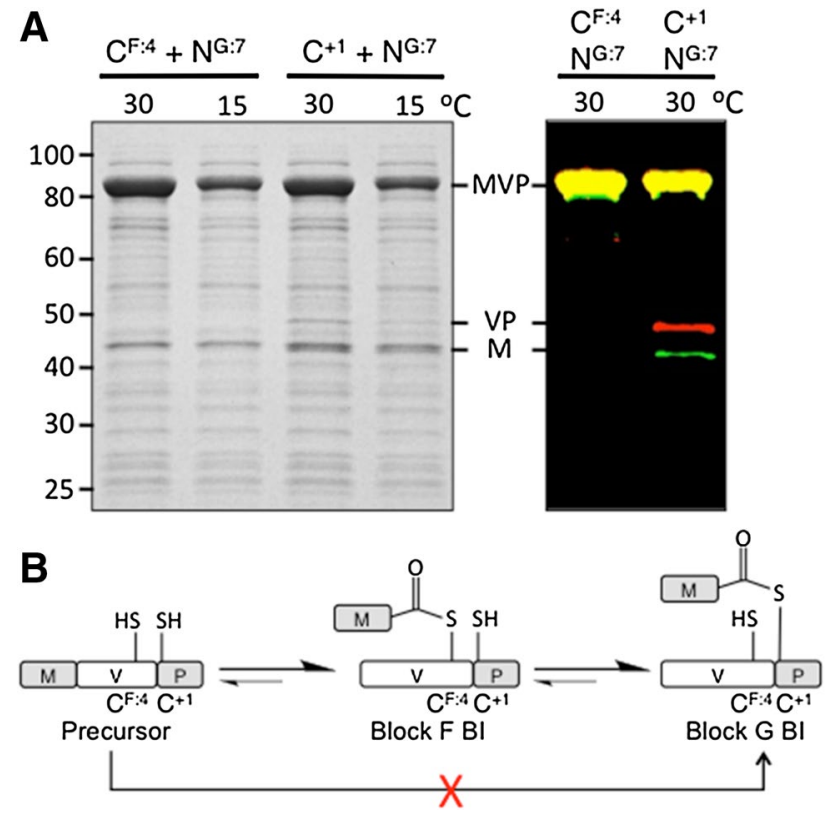

Fig. 5 Analysis of alanine substitutions at conserved cysteines in the Bvi IcmO intein. a Double mutations were made in the MVP precursor substituting alanine for $\mathrm{Asn}^{\mathrm{G}: 7}$ and either $\mathrm{Cys}^{\mathrm{F}: 4}$ or $\mathrm{Cys}^{+1}$. The $\mathrm{Asn}^{\mathrm{G}: 7}$ substitution prevents on-pathway $\mathrm{BI}^{\mathrm{G}}$ resolution. MVP precursors with the indicated residues mutated to alanine were expressed at $30{ }^{\circ} \mathrm{C}$ for $2 \mathrm{~h}$ or $15{ }^{\circ} \mathrm{C}$ overnight. Left panel SDS-PAGE stained with Simply Blue Safe Stain. Right panel Western Blots were probed using IR-dye tagged secondary antibodies with $\mathrm{M}$ shown in green and $\mathrm{P}$ shown in red. Yellow bands indicate an equal reaction with both secondary antibodies. $\mathbf{b}$ The forward and reverse reaction scheme for MVP precursor to $\mathrm{BI}^{\mathrm{G}}$. The red ' $\mathrm{X}$ ' indicates that this reaction was not detected

2011). This study proved that all three inteins splice using the class 3 mechanism as demonstrated by (1) the absolute requirement of $\mathrm{Cys}^{\mathrm{F}: 4}$ for splicing and $\mathrm{N}$-terminal splice junction cleavage, (2) mutation of the class 3 specific signature residue $\operatorname{Trp}^{\mathrm{B}: 12}$ blocked splicing, and (3) the inferred presence of $\mathrm{BI}^{\mathrm{F}}$ by thiol induced decay of purified BIs from MSP and MLP. It is interesting to note that C-terminal cleavage by $\mathrm{Asn}^{\mathrm{G}: 7}$ in all three inteins appears to be strongly coupled to earlier steps in the splicing reaction as observed previously in some other inteins (Martin et al. 2001; Mills et al. 2014; Volkmann and Mootz 2013), since $\mathrm{His}^{\mathrm{B}: 10}, \operatorname{Trp}^{\mathrm{B}: 12}, \mathrm{Cys}^{\mathrm{F}: 4}, \mathrm{Ser}^{+1}$ and $\mathrm{Cys}^{+1}$ mutations did not yield C-terminal splice junction cleavage products.

It is difficult to experimentally distinguish between $\mathrm{BI}^{\mathrm{G}}$ (III, Fig. 1) and $\mathrm{BI}^{\mathrm{F}}$ (IIc) because they have the same mobility in SDS-PAGE, the same mass and have not been detected by Mass Spectrometry. Instead, indirect approaches have been employed to identify BIs, especially the difference in lability of BI thioester vs. ester linkages in vivo and in vitro. Thioester-linked BIs rarely accumulate in E. coli, while ester-linked BIs are often detected (Brace et al. 2010; Mills et al. 2014; Southworth et al. 2000; Tori et al. 2010; Tori and Perler 2011; Volkmann and Mootz 2013; Xu et al. 1994; Xu and Perler 1996). The in vivo instability of thioester linked BIs was observed in this study since only products of BI thiolysis accumulated in MVP (both BIs have a thioester linkage) after $\mathrm{BI}^{\mathrm{G}}$ resolution was prevented by mutation of $\mathrm{Asn}^{\mathrm{G}: 7}$. Based on (thio) ester stability, the MSP and MLP BIs that accumulated in vivo after alanine substitution of $\mathrm{Asn}^{\mathrm{G}: 7}$ are most likely the ester-linked $\mathrm{BI}^{\mathrm{G}}$ rather than the labile thioester linked $\mathrm{BI}^{\mathrm{F}}$ (Fig. 4c). These ester-linked BIs should be stable under the mild conditions used for in vitro thiolysis experiments. However, at $\mathrm{pH} 9$ both the MSP and MLP BIs decayed during DTT treatment. At first glance, these results seem inconsistent because the in vivo data suggest accumulation of the ester linked $\mathrm{BI}^{\mathrm{G}}$, while the in vitro data suggest the presence of the thioester containing $\mathrm{BI}^{\mathrm{F}}$. The reversibility of step 2c (Figs. 1, 5b), explains this conundrum (Brace et al. 2010; Tori et al. 2010; Tori and Perler 2011). The reverse reaction was directly observed when MSP and MLP BIs converted back to linear precursors (Ic) at $\mathrm{pH} 9$ in the absence of DTT (Fig. 4). If DTT is present during the reverse reaction, $\mathrm{BI}^{\mathrm{F}}$ is eliminated by DTT and purified $\mathrm{BI}^{\mathrm{G}}$ continually converts to $\mathrm{BI}^{\mathrm{F}}$ to maintain the equilibrium between the two BIs, eventually resulting in the complete disappearance of both BIs due to thiolysis of $\mathrm{BI}^{\mathrm{F}}$. The observation that DTT was unable to cleave denatured MLP $\mathrm{BI}$ confirms the hypothesis that $\mathrm{BI}^{\mathrm{G}}$ and not $\mathrm{BI}^{\mathrm{F}}$ accumulates in vivo because direct cleavage of $\mathrm{BI}^{\mathrm{G}}$ by thiols would not occur under the experimental conditions (Fig. 4c) and denaturation only prevents forward or reverse reactions while leaving any (thio)ester bond intact.

The Bvi IcmO intein provided a sensitive means of analyzing the mandatory order of BI formation in class 3 inteins. It is possible that $\mathrm{Cys}^{+1}$ can compete with $\mathrm{Cys}^{\mathrm{F}: 4}$ for attack on the peptide bond at the N-terminal splice junction because this residue normally attacks the $\mathrm{N}$-terminal splice junction in other intein classes during steps $2 \mathrm{a}$ or 1b (Eryilmaz et al. 2014; Mills et al. 2014; Southworth et al. 2000; Volkmann and Mootz 2013). Although previous studies with other class 3 inteins demonstrated that $\mathrm{Ser}^{+1}$ cannot initiate the splicing reaction, they all required $\mathrm{Ser}^{+1}$ to attack an N-terminal splice junction that was primed for attack by a cysteine nucleophile and thus may not work because of mechanistic differences caused by the different nucleophiles (Brace et al. 2010; Tori et al. 2010; Tori and Perler 2011). The Bvi IcmO intein allows testing of a naturally occurring $\mathrm{Cys}^{+1}$ in a class 3 intein without the problems associated with changing the nucleophile. $\mathrm{Cys}^{+1} \mathrm{did}$ not generate any $\mathrm{BI}$ or $\mathrm{N}$-terminal splice junction cleavage products in the sensitive fluorescent Western Blot assay of the MVP Cys ${ }^{\mathrm{F}: 4}$ plus Asn ${ }^{\mathrm{G}: 7}$ double mutant under conditions where cleavage products were produced by $\mathrm{Cys}^{\mathrm{F}: 4}$ in the MVP $\mathrm{Cys}^{+1}$ plus Asn $^{\mathrm{G}: 7}$ double mutant. It is unlikely 
that undetected $\mathrm{BI}^{\mathrm{G}}$ formed or decayed in this experiment because the IR dyes used can detect picogram amounts of protein and microgram amounts of MVP precursor were queried. These results demonstrate that (1) the presence of a natural $\mathrm{Cys}^{+1}$ cannot substitute for the loss of $\mathrm{Cys}^{\mathrm{F}: 4}$ by mutation and (2) the order of $\mathrm{BI}$ formation in class 3 inteins must be $\mathrm{BI}^{\mathrm{F}}$ followed by $\mathrm{BI}^{\mathrm{G}}$ (Fig. 5b). This reaction order is likely maintained by local changes at the intein active site after formation of $\mathrm{BI}^{\mathrm{F}}$ that are required to properly align or activate $\mathrm{Cys}^{+1}$ for the next catalytic step.

In summary, this study provides experimental evidence that all three class 3 mini-inteins are active. We conclude that there is no competition between the two catalytic cysteines for attack on the N-terminal splice junction and that the Bvi IcmO intein is unable to splice by the class 2 mechanism. The Bvi IcmO intein expands the repertoire of potential insertion sites for class 3 inteins in target proteins to include Cys for the numerous in vivo and in vitro applications based on intein technology (Aranko et al. 2014; Topilina and Mills 2014; Wood and Camarero 2014). Inteins continue to prove to be intriguing and robust single turnover enzymes.

Acknowledgments This work was funded by New England Biolabs, Inc. We thank Dr. Don Comb (Founder of NEB) for support and encouragement. We thank Dr. James Tiedje (Michigan State University) for kindly providing Burkholderia vietnamiensis G4 genomic DNA.

Open Access This article is distributed under the terms of the Creative Commons Attribution 4.0 International License (http://creativecommons.org/licenses/by/4.0/), which permits unrestricted use, distribution, and reproduction in any medium, provided you give appropriate credit to the original author(s) and the source, provide a link to the Creative Commons license, and indicate if changes were made.

\section{References}

Amitai G, Callahan BP, Stanger MJ, Belfort G, Belfort M (2009) Modulation of intein activity by its neighboring extein substrates. Proc Natl Acad Sci USA 106:11005-11010

Aranko AS, Wlodawer A, Iwai H (2014) Nature's recipe for splitting inteins. Protein Eng Des Sel 27:263-271

Barzel A, Naor A, Privman E, Kupiec M, Gophna U (2011) Homing endonucleases residing within inteins: evolutionary puzzles awaiting genetic solutions. Biochem Soc Trans 39:169-173

Brace LE, Southworth MW, Tori K, Cushing ML, Perler F (2010) The Deinococcus radiodurans Snf2 intein caught in the act: detection of the Class 3 intein signature Block F branched intermediate. Protein Sci 19:1525-1533

Cheriyan M, Pedamallu CS, Tori K, Perler F (2013) Faster protein splicing with the Nostoc punctiforme DnaE intein using nonnative extein residues. J Biol Chem 288:6202-6211

Cheriyan M, Chan SH, Perler F (2014) Traceless splicing enabled by substrate-induced activation of the Nostoc punctiforme $\mathrm{Npu}$ DnaE intein after mutation of a catalytic cysteine to serine. J Mol Biol 426:4018-4029
Chong S et al (1997) Single-column purification of free recombinant proteins using a self-cleavable affinity tag derived from a protein splicing element. Gene 192:271-281

Dalgaard JZ, Moser MJ, Hughey R, Mian IS (1997) Statistical modeling, phylogenetic analysis and structure prediction of a protein splicing domain common to inteins and hedgehog proteins. J Comput Biol 4:193-214

Eiglmeier K, Honore N, Woods SA, Caudron B, Cole ST (1993) Use of an ordered cosmid library to deduce the genomic organization of Mycobacterium leprae. Mol Microbiol 7:197-206

Eryilmaz E, Shah NH, Muir TW, Cowburn D (2014) Structural and dynamical features of inteins and implications on protein splicing. J Biol Chem 289:14506-14511

Iwai H, Zuger S, Jin J, Tam PH (2006) Highly efficient protein transsplicing by a naturally split DnaE intein from Nostoc punctiforme. FEBS Lett 580:1853-1858

Kawasaki M, Nogami S, Satow Y, Ohya Y, Anraku Y (1997) Identification of three core regions essential for protein splicing of the yeast Vma1 protozyme. A random mutagenesis study of the entire Vma1-derived endonuclease sequence. J Biol Chem 272:15668-15674

Liu Z, Frutos S, Bick MJ, Vila-Perello M, Debelouchina GT, Darst SA, Muir TW (2014) Structure of the branched intermediate in protein splicing. Proc Natl Acad Sci USA 111:8422-8427

Martin DD, Xu MQ, Evans TC Jr (2001) Characterization of a naturally occurring trans-splicing intein from Synechocystis sp. PCC6803. Biochemistry 40:1393-1402

Mills KV, Johnson MA, Perler FB (2014) Protein splicing: how inteins escape from precursor proteins. J Biol Chem 289:14498-14505

Mohan A, Padiadpu J, Baloni P, Chandra N (2015) Complete Genome Sequences of a Mycobacterium smegmatis Laboratory Strain (MC2 155) and Isoniazid-Resistant (4XR1/R2) Mutant Strains. Genome Announc 3(1):e01520-14. doi:10.1128/ genomeA.01520-14

Muona M, Aranko AS, Raulinaitis V, Iwai H (2010) Segmental isotopic labeling of multi-domain and fusion proteins by protein trans-splicing in vivo and in vitro. Nat Protoc 5:574-587

Nordberg H et al (2014) The genome portal of the Department of Energy Joint Genome Institute: 2014 updates. Nucleic Acids Res 42:D26-D31

Novikova O, Topilina N, Belfort M (2014) Enigmatic distribution, evolution, and function of inteins. J Biol Chem 289:14490-14497

Perler FB (2002) InBase: the intein database. Nucleic Acids Res 30:383-384

Perler FB et al (1994) Protein splicing elements: inteins and exteinsa definition of terms and recommended nomenclature. Nucleic Acids Res 22:1125-1127

Perler FB, Olsen GJ, Adam E (1997) Compilation and analysis of intein sequences. Nucleic Acids Res 25:1087-1093

Pietrokovski S (1994) Conserved sequence features of inteins (protein introns) and their use in identifying new inteins and related proteins. Protein Sci 3:2340-2350

Pietrokovski S (1998) Modular organization of inteins and C-terminal autocatalytic domains. Protein Sci 7:64-71

Romanelli A, Shekhtman A, Cowburn D, Muir TW (2004) Semisynthesis of a segmental isotopically labeled protein splicing precursor: NMR evidence for an unusual peptide bond at the N-exteinintein junction. Proc Natl Acad Sci USA 101:6397-6402

Southworth MW, Amaya K, Evans TC, Xu MQ, Perler FB (1999) Purification of proteins fused to either the amino or carboxy terminus of the Mycobacterium xenopi gyrase A intein. Biotechniques 27:110-114 (116, 118-120)

Southworth MW, Benner J, Perler FB (2000) An alternative protein splicing mechanism for inteins lacking an N-terminal nucleophile. EMBO J 19:5019-5026 
Topilina NI, Mills KV (2014) Recent advances in in vivo applications of intein-mediated protein splicing. Mob DNA 5:5

Tori K, Perler FB (2011) Expanding the definition of class 3 inteins and their proposed phage origin. J Bacteriol 193:2035-2041

Tori K et al (2010) Splicing of the Mycobacteriophage Bethlehem DnaB intein: identification of a new mechanistic class of inteins that contain an obligate block $\mathrm{F}$ nucleophile. J Biol Chem 285:2515-2526

Volkmann G, Mootz HD (2013) Recent progress in intein research: from mechanism to directed evolution and applications. Cell Mol Life Sci 70:1185-1206
Wood DW, Camarero JA (2014) Intein applications: from protein purification and labeling to metabolic control methods. J Biol Chem 289:14512-14519

Wu Q, Gao Z, Wei Y, Ma G, Zheng Y, Dong Y, Liu Y (2014) Conserved residues that modulate protein trans-splicing of $\mathrm{Npu}$ DnaE split intein. Biochem J 461:247-255

Xu MQ, Perler FB (1996) The mechanism of protein splicing and its modulation by mutation. EMBO J 15:5146-5153

Xu MQ, Comb DG, Paulus H, Noren CJ, Shao Y, Perler FB (1994) Protein splicing: an analysis of the branched intermediate and its resolution by succinimide formation. EMBO J 13:5517-5522 